\title{
Application of Markowitz Model to Mongolian Government Budget
}

\author{
Ch. Ankhbayar, B. Lkhagvajav, N. Tungalag, R. Enkhbat \\ Business School, National University of Mongolia, Ulaanbaatar, Mongolia \\ Email: enkhbatm@nun.edu.mn,renkhbat46@yahoo.com
}

How to cite this paper: Ankhbayar, Ch. Lkhagvajav, B., Tungalag, N. and Enkhbat, R. (2019) Application of Markowitz Model to Mongolian Government Budget. iBusiness, 11, 42-50.

https://doi.org/10.4236/ib.2019.113004

Received: May 17, 2019

Accepted: September 16, 2019

Published: September 19, 2019

Copyright ( 2019 by author(s) and Scientific Research Publishing Inc. This work is licensed under the Creative Commons Attribution International License (CC BY 4.0).

http://creativecommons.org/licenses/by/4.0/

\begin{abstract}
We apply Markowitz portfolio theory to Mongolian economy in order to define optimal budget structure. We assume that the government revenue is a portfolio consisting of seven major taxes and non-tax revenues. We minimize the variance of the portfolio under fixed return of the government revenue. This optimization problem has been solved by the conditional gradient method on MATLAB. Computational results based on Mongolian economic data are provided.
\end{abstract}

Keywords

Markowitz Model

\section{Introduction}

Financial portfolio optimization is widely used in mathematics, statistics, economics and engineering. Fundamental breakthrough in the problem of asset allocation and portfolio optimization is dated to Markowitz's Modern Portfolio Theory [1]. It considers rational investors and models with the problem of minimizing the mean-variance of the portfolio with a fixed value for the expected return on the entire portfolio. The model also assumes a market without any taxes or transaction costs, and where short selling is disallowed but assets are infinitely divisible and can be traded with any non-negative fractions.

There are many works devoted to optimization methods and algorithms for solving the portfolio variance minimization problem. This problem belongs to the convex optimization problem so any stationary point found by an optimization method provides a global solution to the problem. Also, the Markowitz model has been extended in various ways in the literature [2]-[13]. Tobin James's work [9] considers the inclusion of risk-free assets in Markowitz model by the devel- 
opment of the Separation theorem which states that in the presence of a risk-free asset, the optimal risky portfolio can be obtained without any knowledge of the investor's preferences.

Sharpe's Capital Asset Pricing Model (CAPM) [14] takes into account the asset's sensitivity to non-diversifiable risk while it is being added to an already existing well-diversified portfolio. It considers the importance of the covariance structure of the returns, the variance of the portfolio and the market premium. The model assumes that the investors are rational and risk-averse, are broadly diversified across a range of investments, and that they cannot influence the prices of the assets. Assumptions regarding trade or transaction costs, short-selling and trades with non-negative fractions do apply from the traditional Markowitz's framework.

Considering the equity markets in perspective, Fernholzs Stochastic Portfolio Theory [2] discusses a descriptive theory that provides a framework for analyzing portfolio behavior and equity market structure that has both theoretical and practical applications.

Portfolio optimization problems have been studied in [3] [12] [15] [16] and [17]. Formulation of Markowitz's portfolio optimization problem is viewed as a quadratic optimization problem. [10] and [18] provides comprehensive literature to convex and numerical optimization methods to solve such a formulation.

[19] explores a global optimization approach to scenario generation and portfolio optimization looking at them as individual problems. [12] proposes a stochastic programming approach for multi-period portfolio optimization. [5] presents a multi-period scenario generation approach to support portfolio optimization and [20] discusses scenario generation, mathematical models and algorithms for the portfolio optimization problem. [21] explores portfolio selection using hierarchical Bayesian analysis and Markov Chain Monte Carlo (MCMC) methods. [4] discusses the portfolio optimization with an envelope-based multi-objective evolutionary algorithm with a variety of non-convex constraints.

[22] solves the portfolio optimization problem using genetic algorithm. [23] applies genetic algorithms in a multi-stage portfolio optimization system. [24] solves the problem with the same method taking into account transaction costs and minimum transaction lot constraints.

[25] examines constrained Markowitz portfolio selection using ant colony optimization. [26] considers multi-objective particle swarm optimization approach to the portfolio optimization problem. In this paper, for solving the variance minimization problem, we use the conditional gradient method [18] which uses a series of linear programming problems. The paper is organized as follows. In Methodology Section, we introduce briefly Markowitz portfolio theory and show how to apply the theory to Mongolian government budget. In Data Description Section, we use Mongolian economic data and construct matrix tables for the proposed model. In the last section, we implement Markowitz model for Mongolian government budget. 


\section{Methodology}

Assume that a government revenue consists of $n$ revenues

$$
A=\sum_{i=1}^{n} A_{i}
$$

where $A$ is a total government revenue, and $A_{i}$ is $i$-th type of revenue, $i=1,2, \cdots, n$.

We can consider $A$ as a portfolio of $n$ assets with weights $x_{i}$ which means $A_{i}=x_{i} A, i=1,2, \cdots, n$.

Clearly,

$$
\sum_{i=1}^{n} x_{i}=1, \quad x_{i} \geq 0, \quad i=1,2, \cdots, n .
$$

Let $r_{1}, r_{2}, \cdots, r_{n}$ be rates of the tax revenues returns.

These have expected values

$$
E\left(r_{1}\right)=\bar{r}_{1}, E\left(r_{2}\right)=\bar{r}_{2}, \cdots, E\left(r_{n}\right)=\bar{r}_{n} .
$$

Then the rate of return of the portfolio is

$$
r=\sum_{i=1}^{n} x_{i} r_{i}
$$

We denote the variance of the return of $i$-th tax revenue by $\sigma_{i}^{2}$, the variance of the return of the portfolio by $\sigma^{2}$, and the covariance of the return of $i$-th revenue with $j$-th revenue by $\sigma_{i j}$. It is well known that [1] [27]

$$
\sigma^{2}=\sum_{i=1}^{n} \sum_{j=1}^{n} x_{i} x_{j} \sigma_{i j}
$$

To find a minimum-variance portfolio, we fix the mean value at same arbitrary value $\bar{r}$. Then we find the optimal portfolio by solving the following minimization problem [1] [27]:

$$
\min \frac{1}{2} \sum_{i=1}^{n} \sum_{j=1}^{n} x_{i} x_{j} \sigma_{i j}
$$

subject to

$$
\begin{gathered}
\sum_{i=1}^{n} x_{i} \bar{r}_{i}=\bar{r} \\
\sum_{i=1}^{n} x_{i}=1 \\
x_{i} \geq 0, \quad i=1,2, \cdots, n
\end{gathered}
$$

Note that problem (1)-(4) is convex from a view point of optimization theory. It can be checked that the matrix of covariance $C_{n \times n}=\left(\sigma_{i j}\right)$ is positive defined. In order to find a solution to problem (1)-(4), we need to write the Lagrangian as

$$
L=\frac{1}{2} \sum_{i=1}^{n} \sum_{j=1}^{n} x_{i} x_{j} \sigma_{i j}+\lambda_{1}\left(\sum_{i=1}^{n} x_{i} \bar{r}_{i}-\bar{r}\right)+\lambda_{2}\left(\sum_{i=1}^{n} x_{i}-1\right)+\sum_{i=1}^{n} \mu_{i} x_{i}
$$

taking into account condition (4). 
Then if we apply Karush-Kuhn-Tucker optimality condition to problem (1)-(4), we have

$$
\left\{\begin{array}{l}
\frac{\partial L}{\partial x_{i}}=\sum_{i=1}^{n} \sigma_{i j} x_{j}+\lambda_{1} \bar{r}_{i}+\lambda_{2}+\mu_{i}=0, i=1,2, \cdots, n \\
\mu_{i} x_{i}=0, \quad i=1,2, \cdots, n \\
\lambda_{1}^{2}+\lambda_{2}^{2}+\sum_{i=1}^{n} \mu_{i}^{2}>0, \quad \mu_{i} \geq 0, \quad i=1,2, \cdots, n
\end{array}\right.
$$

To find an optimal solution, we combine system (5) with (2)-(4). It means that

$$
\left\{\begin{array}{l}
\sum_{i=1}^{n} \sigma_{i j} x_{j}+\lambda_{1} \bar{r}_{i}+\lambda_{2}+\mu_{i}=0, i=1,2, \cdots, n \\
\sum_{i=1}^{n} x_{i} \bar{r}_{i}=\bar{r} \\
\sum_{i=1}^{n} x_{i}=1 \\
\mu_{i} x_{i}=0, \quad i=1,2, \cdots, n \\
\mu_{i} \geq 0, \quad i=1,2, \cdots, n
\end{array}\right.
$$

This nonlinear system has $(3 n+2)$ linear and nonlinear equations with $(2 n+2)$ unknowns. So it is better to solve problem (1)-(4) by convex optimization methods and algorithm. For instance, it is convenient to solve problem (1)-(4) by conditional gradient method [27] since at each iteration of the algorithm we solve just a linear programming problem.

\section{Data Description}

For numerical analysis we use the following Mongolian economic data for period 1991-2018 which shows structure of government revenue consisted of tax and nontax revenues (Tables 1-3).

Table 1. Weight of government revenue.

\begin{tabular}{cccccccc}
\hline & $X_{1}$ & $X_{2}$ & $X_{3}$ & $X_{4}$ & $X_{5}$ & $X_{6}$ & $X_{7}$ \\
\hline Year & Income tax $\begin{array}{c}\text { Social security } \\
\text { contributions }\end{array}$ & $\begin{array}{c}\text { Property } \\
\text { taxes }\end{array}$ & $\begin{array}{c}\text { Taxes on domestic } \\
\text { goods \& services }\end{array}$ & $\begin{array}{c}\text { Taxes on } \\
\text { foreign trade }\end{array}$ & $\begin{array}{c}\text { Other } \\
\text { taxes }\end{array}$ & $\begin{array}{c}\text { Non-tax } \\
\text { revenue }\end{array}$ \\
\hline 1991 & 0.358 & 0.099 & 0.001 & 0.301 & 0.041 & 0.013 & 0.187 \\
1992 & 0.427 & 0.071 & 0.000 & 0.243 & 0.113 & 0.015 & 0.131 \\
1993 & 0.493 & 0.049 & 0.000 & 0.245 & 0.114 & 0.011 & 0.087 \\
1994 & 0.372 & 0.073 & 0.000 & 0.227 & 0.088 & 0.024 & 0.217 \\
1995 & 0.336 & 0.109 & 0.000 & 0.194 & 0.066 & 0.024 & 0.270 \\
1996 & 0.280 & 0.113 & 0.000 & 0.229 & 0.085 & 0.035 & 0.258 \\
1997 & 0.281 & 0.095 & 0.000 & 0.284 & 0.040 & 0.036 & 0.263 \\
1998 & 0.173 & 0.109 & 0.001 & 0.321 & 0.006 & 0.032 & 0.358 \\
1999 & 0.147 & 0.112 & 0.001 & 0.352 & 0.034 & 0.034 & 0.320 \\
\hline
\end{tabular}




\section{Continued}

\begin{tabular}{|c|c|c|c|c|c|c|c|}
\hline 2000 & 0.207 & 0.108 & 0.001 & 0.347 & 0.062 & 0.032 & 0.244 \\
\hline 2001 & 0.147 & 0.123 & 0.004 & 0.379 & 0.062 & 0.033 & 0.253 \\
\hline 2002 & 0.152 & 0.114 & 0.007 & 0.374 & 0.052 & 0.054 & 0.247 \\
\hline 2003 & 0.176 & 0.118 & 0.008 & 0.343 & 0.059 & 0.056 & 0.240 \\
\hline 2004 & 0.202 & 0.115 & 0.008 & 0.343 & 0.063 & 0.087 & 0.182 \\
\hline 2005 & 0.213 & 0.114 & 0.008 & 0.323 & 0.068 & 0.100 & 0.174 \\
\hline 2006 & 0.351 & 0.082 & 0.005 & 0.259 & 0.053 & 0.079 & 0.171 \\
\hline 2007 & 0.345 & 0.085 & 0.004 & 0.219 & 0.054 & 0.091 & 0.201 \\
\hline 2008 & 0.348 & 0.106 & 0.004 & 0.259 & 0.065 & 0.090 & 0.129 \\
\hline 2009 & 0.261 & 0.132 & 0.006 & 0.255 & 0.058 & 0.101 & 0.187 \\
\hline 2010 & 0.312 & 0.106 & 0.004 & 0.277 & 0.062 & 0.099 & 0.139 \\
\hline 2011 & 0.197 & 0.112 & 0.004 & 0.339 & 0.080 & 0.135 & 0.132 \\
\hline 2012 & 0.179 & 0.138 & 0.004 & 0.337 & 0.067 & 0.136 & 0.139 \\
\hline 2013 & 0.187 & 0.147 & 0.007 & 0.323 & 0.064 & 0.125 & 0.146 \\
\hline 2014 & 0.175 & 0.146 & 0.008 & 0.297 & 0.057 & 0.138 & 0.178 \\
\hline 2015 & 0.196 & 0.174 & 0.014 & 0.275 & 0.054 & 0.147 & 0.139 \\
\hline 2016 & 0.173 & 0.195 & 0.017 & 0.327 & 0.054 & 0.097 & 0.137 \\
\hline 2017 & 0.222 & 0.182 & 0.018 & 0.296 & 0.070 & 0.081 & 0.132 \\
\hline 2018 & 0.226 & 0.176 & 0.015 & 0.321 & 0.074 & 0.077 & 0.111 \\
\hline
\end{tabular}

Source: National Statistical Office, https://www.1212.mn/.

Table 2. Government revenue growth.

\begin{tabular}{cccccccc}
\hline Year & $\begin{array}{c}\text { Income } \\
\text { tax }\end{array}$ & $\begin{array}{c}\text { Social security } \\
\text { contributions }\end{array}$ & $\begin{array}{c}\text { Property } \\
\text { taxes }\end{array}$ & $\begin{array}{c}\text { Taxes on domestic } \\
\text { goods \& services }\end{array}$ & $\begin{array}{c}\text { Taxes on } \\
\text { foreign trade }\end{array}$ & $\begin{array}{c}\text { Other } \\
\text { taxes }\end{array}$ & $\begin{array}{c}\text { Non-tax } \\
\text { revenue }\end{array}$ \\
\hline 1992 & 1.117 & 0.277 & 0.000 & 0.436 & 3.917 & 1.010 & 0.247 \\
1993 & 4.194 & 2.125 & 0.017 & 3.531 & 3.540 & 2.497 & 1.986 \\
1994 & 0.127 & 1.210 & 3.918 & 0.381 & 0.146 & 2.173 & 2.711 \\
1995 & 0.515 & 1.512 & 0.800 & 0.439 & 0.269 & 0.681 & 1.094 \\
1996 & -0.060 & 0.172 & -0.174 & 0.325 & 0.454 & 0.633 & 0.073 \\
1997 & 0.373 & 0.150 & 0.758 & 0.700 & -0.368 & 0.398 & 0.395 \\
1998 & -0.338 & 0.227 & 2.064 & 0.215 & -0.828 & -0.019 & 0.469 \\
1999 & -0.059 & 0.143 & 0.246 & 0.221 & 4.973 & 0.178 & -0.009 \\
2000 & 0.898 & 0.299 & -0.036 & 0.324 & 1.475 & 0.259 & 0.025 \\
2001 & -0.129 & 0.395 & 4.949 & 0.338 & 0.211 & 0.264 & 0.271 \\
2002 & 0.123 & 0.008 & 0.951 & 0.073 & -0.090 & 0.768 & 0.061 \\
2003 & 0.347 & 0.199 & 0.372 & 0.065 & 0.328 & 0.194 & 0.128 \\
2004 & 0.477 & 0.259 & 0.249 & 0.285 & 0.370 & 1.017 & -0.022 \\
2005 & 0.239 & 0.165 & 0.102 & 0.109 & 0.274 & 0.348 & 0.120 \\
\hline
\end{tabular}




\begin{tabular}{llllllll}
\multicolumn{2}{l}{ Continued } & \multicolumn{7}{l}{0.285} & 0.595 \\
\hline 2006 & 1.671 & 0.171 & 0.092 & 0.302 & 0.265 & 0.285 \\
2007 & 0.360 & 0.434 & 0.195 & 0.167 & 0.422 & 0.586 & 0.628 \\
2008 & 0.164 & 0.429 & 0.114 & 0.365 & 0.374 & 0.140 & -0.261 \\
2009 & -0.311 & 0.149 & 0.213 & -0.095 & -0.176 & 0.032 & 0.336 \\
2010 & 0.874 & 0.257 & 0.238 & 0.701 & 0.667 & 0.541 & 0.163 \\
2011 & -0.145 & 0.429 & 0.242 & 0.658 & 0.745 & 0.848 & 0.287 \\
2012 & 0.045 & 0.424 & 0.279 & 0.145 & -0.030 & 0.164 & 0.213 \\
2013 & 0.273 & 0.297 & 1.005 & 0.169 & 0.165 & 0.116 & 0.279 \\
2014 & -0.007 & 0.050 & 0.139 & -0.029 & -0.068 & 0.167 & 0.291 \\
2015 & 0.063 & 0.132 & 0.725 & -0.120 & -0.098 & 0.012 & -0.258 \\
2016 & -0.109 & 0.132 & 0.210 & 0.205 & 0.025 & -0.332 & -0.004 \\
2017 & 0.546 & 0.124 & 0.253 & 0.088 & 0.560 & 0.001 & 0.160 \\
2018 & 0.293 & 0.227 & 0.078 & 0.378 & 0.332 & 0.214 & 0.071 \\
\hline & & & & & & & \\
\hline
\end{tabular}

Table 3. Covariance matrix of government revenue.

\begin{tabular}{cccccccc}
\hline COVAR $(X)$ & $X_{1}$ & $X_{2}$ & $X_{3}$ & $X_{4}$ & $X_{5}$ & $X_{6}$ & $X_{7}$ \\
\hline$X_{1}$ & 0.7692 & 0.2684 & -0.2538 & 0.5033 & 0.5707 & 0.3391 & 0.2542 \\
$X_{2}$ & 0.2684 & 0.2266 & 0.1035 & 0.2415 & 0.1775 & 0.2269 & 0.2444 \\
$X_{3}$ & -0.2538 & 0.1035 & 1.4036 & -0.0608 & -0.3865 & 0.1362 & 0.3116 \\
$X_{4}$ & 0.5033 & 0.2415 & -0.0608 & 0.4410 & 0.4042 & 0.2934 & 0.2267 \\
$X_{5}$ & 0.5707 & 0.1775 & -0.3865 & 0.4042 & 1.7830 & 0.3059 & 0.0925 \\
$X_{6}$ & 0.3391 & 0.2269 & 0.1362 & 0.2934 & 0.3059 & 0.3925 & 0.3184 \\
$X_{7}$ & 0.2542 & 0.2444 & 0.3116 & 0.2267 & 0.0925 & 0.3184 & 0.4095 \\
\hline
\end{tabular}

\section{Numerical Results}

In this section, we implement the Markowitz model for Mongolian economy. We examine government budget revenue structure which depends on seven types of tax and nontax revenues.

Variable $x_{i}$ is the weight of $i$-th tax revenue in the portfolio. The Mongolian government budget consists of the following revenues such as income tax, social security contributions, property taxes, taxes on domestic goods and services, taxes on foreign trade, other taxes and non-tax revenues. Table 4 shows the initial values of variables as well as the optimal solution of problem (1)-(4) found by the conditional gradient method on MATLAB.

Thus, the government should take into account these results in fiscal policy decision making. 
Table 4. Solution.

\begin{tabular}{cccc}
\hline Name & Initial value & Optimal value & Change \\
\hline Income tax & 0.255 & 0.227 & $-2.8 \%$ \\
Social security contributions & 0.118 & 0.115 & $-0.3 \%$ \\
Property taxes & 0.005 & 0.018 & $1.3 \%$ \\
Taxes on domestic goods \& services & 0.296 & 0.194 & $-10.2 \%$ \\
Taxes on foreign trade & 0.063 & 0.040 & $-2.3 \%$ \\
Other taxes & 0.071 & 0.147 & $7.6 \%$ \\
Non-tax revenue & 0.192 & 0.260 & $6.8 \%$ \\
\hline
\end{tabular}

\section{Conclusion}

We have tested the Markowitz model on Mongolian economic data in order to define optimal structure of the government revenue which consists of $7 \mathrm{com}$ ponents. Since the variance minimization problem was convex quadratic, for solving the problem we have applied the conditional gradient method coded in MATLAB. The numerical solution was obtained. In the same way, we can consider the problem of maximizing the government return subject to variance constraint. But it will be discussed in the next paper.

\section{Acknowledgements}

This work was supported by the research grant P2018-3588 of National University of Mongolia.

\section{Conflicts of Interest}

The authors declare no conflicts of interest regarding the publication of this paper.

\section{References}

[1] Markowitz, H. (1952) Portfolio Selection. The Journal of Finance, 7, 77-91. https://doi.org/10.1111/j.1540-6261.1952.tb01525.x

[2] Fernholz, R.E. (2002) Stochastic Portfolio Theory, Volume 48. Springer Verlag, New York. https://doi.org/10.1007/978-1-4757-3699-1

[3] Homan, M., Brochu, E. and de Freitas, N. (2011) Portfolio Allocation for Bayesian Optimization. In: Heckerman, D. and Mamdani, A., Eds., Uncertainty in Artificial Intelligence, Elsevier, ¡Amsterdam, 327-336.

[4] Branke, J., Scheckenbach, B., Stein, M., Deb, K. and Schmeck, H. (2009) Portfolio Optimization with an Envelope-Based Multi-Objective Evolutionary Algorithm. European Journal of Operational Research, 199, 684-693. https://doi.org/10.1016/j.ejor.2008.01.054

[5] Deniz, E. (2009) Multi-Period Scenario Generation to Support Portfolio Optimization. PhD Thesis, Rutgers, The State University of New Jersey, New Brunswick, NJ.

[6] Daly, J., Crane, M. and Ruskin, H.J. (2008) Random Matrix Theory Filters in Portfolio Optimisation: A Stability and Risk Assessment. Physica A: Statistical Mechan- 
ics and Its Applications, 387, 4248-4260.

https://doi.org/10.1016/j.physa.2008.02.045

[7] Christine Strauss (2001) Ant Colony Optimization in Multi Objective Portfolio Selection. 4th Metaheuristics International Conference, Porto, Portugal, 16-20 July 2001, 243-248.

[8] Geyer, A., Hanke, M. and Weissensteiner, A. (2009) A Stochastic Programming Approach for Multi-Period Portfolio Optimization. Computational Management Science, 6, 187-208. https://doi.org/10.1007/s10287-008-0089-9

[9] Hester, D.D. and James, T. (1967) Risk Aversion and Portfolio Choice. John Wiley and Sons, Inc., New York.

[10] Nocedal, J. and Wright, S.J. (1999) Numerical Optimization. Springer Series in Operations Research. 2nd Edition, Springer, Berlin. https://doi.org/10.1007/b98874

[11] Chen, W., Zhang, R.-T., Cai, Y.-M. and Xu, F.-S. (2006) Particle Swarm Optimization for Constrained Portfolio Selection Problems. 2006 International Conference on Machine Learning and Cybernetics, Dalian, 13-16 August 2006, 2425-2429. https://doi.org/10.1109/ICMLC.2006.258773

[12] Black, F. and Litterman, R. (1992) Global Portfolio Optimization. Financial Analysts Journal, 48, 28-43. https://doi.org/10.2469/faj.v48.n5.28

[13] Bolshakova, I., Girlich, E. and Kovalev, M. (2009) Portfolio Optimization Problems: A Survey. Otto-von-Guericke University Magdeburg, Germany.

[14] Sharpe, W.F. (1964) Capital Asset Prices: A Theory of Market Equilibrium under Conditions of Risk. The Journal of Finance, 19, 425-442. https://doi.org/10.1111/j.1540-6261.1964.tb02865.x

[15] Christodoulakis, G.A. (2002) Bayesian Optimal Portfolio Selection: The Black-Litterman Approach. Notes for Quantitative Asset Pricing, MSc. Mathematical Trading and Finance, City, University of London, London.

[16] Walters, J. (2009) The Black-Litterman Model in Detail. Harvard Management Company, Boston, MA, 16-67.

[17] Zhou, G. (2009) Beyond Black-Litterman: Letting the Data Speak. The Journal of Portfolio Management, 36, 36-45. https://doi.org/10.3905/JPM.2009.36.1.036

[18] Boyd, S. and Vandenberghe, L. (2009) Convex Optimization. 7th Edition, Cambridge University Press, Cambridge.

[19] Parpas, P., Rustem, B., Wieland, V. and Zakovie, S. (2006) Mean Variance Optimization of Non-Linear Systems and Worst-Case Analysis. Computational Optimization and Applications, 43, 235-259. https://doi.org/10.1007/s10589-007-9136-7

[20] Guastaroba, G., Mitra, G. and Speranza, M.G. (2011) Investigating the Effectiveness of Robust Portfolio Optimization Techniques. Journal of Asset Management, 12, 260-280. https://doi.org/10.1057/jam.2011.7

[21] Greyserman, A., Jones, D. and Strawderman, W. (2006) Portfolio Selection Using Hierarchical Bayesian Analysis and MCMC Methods. Journal of Banking \& Finance, 30, 669-678. https://doi.org/10.1016/j.jbankfin.2005.04.008

[22] Roudier, F. (2006) Portfolio Optimization and Genetic Algorithms. Master's Thesis, Swiss Federal Institute of Technology (ETM), Zurich.

[23] Chan, M.C., Wong, C.C., Cheung, B.K.S. and Tang, G.Y.N. (2002) Genetic Algorithms in Multi-Stage Portfolio Optimization System. Proceedings of the 8 th International Conference of the Society for Computational Economics, Computing in Economics and Finance, Aix-en-Provence, France, 27-29 June 2002, 1-15. 
[24] Lin, D. and Li, X. (2005) A Genetic Algorithm for Solving Portfolio Optimization Problems with Transaction Costs and Minimum Transaction Lots. In: Wang, L., Chen, K. and Ong, Y., Eds., Advances in Natural Computation, Springer, Berlin, Heidelberg, 808-811. https://doi.org/10.1007/11539902_99

[25] Thong, V. (2007) Constrained Markowitz Portfolio Selection Using Ant Colony Optimization. Erasmus University, Rotterdam.

[26] Mishra, S.K., Panda, G. and Meher, S. (2009) Multi-Objective Particle Swarm Optimization Approach to Portfolio Optimization. 2009 World Congress on Nature and Biologically Inspired Computing, Coimbatore, India, 9-11 December 2009, 1612-1615. https://doi.org/10.1109/NABIC.2009.5393659

[27] Markowitz, H.M. (2010) Portfolio Theory: As I Still See It. Annual Review of Financial Economics, 2, 1-23. https://doi.org/10.1146/annurev-financial-011110-134602 